\title{
Tendências da temperatura anual no estado do Tocantins
}

\author{
Roberta Araújo SILVA ${ }^{1 *}$, Eduardo Silvas REIS ${ }^{1}$, Girlene Figueiredo MACIEL ${ }^{1}$ \\ ${ }^{1}$ Universidade Federal de Tocantins, Palms, TO, Brasil. \\ (ORCID: 0000-0002-9682-3006*; 0000-0002-3869-6635; 0000-0001-9217-3731) \\ *E-mail: roberta.as@uft.br (0000-0002-9682-3006)
}

Recebido em 19/06/2019; Aceito em 03/08/2020; Publicado em 26/08/2020.

\begin{abstract}
RESUMO: Neste trabalho foram investigadas as tendências anuais de temperatura absoluta do ar máxima e mínima de seis estações meteorológicas convencionais do Instituto Nacional de Meteorologia - INMET, localizadas no estado do Tocantins, para o período de 1961 a 2017. A análise de tendência foi realizada aplicando o teste não paramétrico de Mann-Kendall. Os resultados indicam tendência significativa de aumento da temperatura máxima anual em todas as estações. A temperatura mínima anual também apresentou tendência positiva, porém com significância estatística apenas para as estações de Porto Nacional, Palmas e Taguatinga. Embora haja diferenças nas tendências entre as estações, verificou-se um aumento sistemático da temperatura máxima e mínima, especialmente a partir da década de 90 . A maior taxa de crescimento da temperatura foi registrada na estação de Palmas, de $4,14^{\circ} \mathrm{C}$ para a mínima e de $3,68{ }^{\circ} \mathrm{C}$ para a máxima, em um período de 23 anos. O aumento da temperatura mínima encontrados nesse trabalho evidencia que essas cidades estão passando por um processo de maior retenção de energia na forma de calor sensível durante a noite, possivelmente devido a substituição da cobertura da superfície terrestre, devido a maior dificuldade da troca de energia entre a superfície e a atmosfera.
\end{abstract}

Palavras-chave: Mann-Kendall; detecção de tendências; mudanças climáticas.

\section{Annual temperature trends in the state of Tocantins}

\begin{abstract}
In this work, the annual trends in absolute and maximum air temperature of six conventional meteorological stations of the National Institute of Meteorology - INMET, located in the state of Tocantins, for the period from 1961 to 2017 were investigated. The trend analysis was performed using the Mann-Kendall non-parametric test. The results indicate a significant trend of increasing the maximum annual temperature in all seasons. The annual minimum temperature also showed a positive trend, but with statistical significance only for the Porto Nacional, Palmas and Taguatinga stations. Although there are differences in trends between seasons, there was a systematic increase in maximum and minimum temperature, especially from the $90 \mathrm{~s}$. The highest rate of temperature growth was registered at Palmas station, from $4.14^{\circ} \mathrm{C}$ to the minimum and $3.68^{\circ}$ $\mathrm{C}$ for the maximum, over a period of 23 years. The increase in the minimum temperature found in this work shows that these cities are going through a process of greater energy retention in the form of sensitive heat during the night, possibly due to the replacement of the Earth's surface coverage, due to the greater difficulty in exchanging energy between the surface and the atmosphere.
\end{abstract}

Keywords: Mann-Kendall; trend detection; climate changes.

\section{INTRODUÇÃO}

As alterações climáticas são uma das questões ambientais mais graves das últimas décadas, pois suas implicações afetam direta e indiretamente as atividades humanas (IPCC, 2007). Esse cenário tem despertado o interesse da comunidade científica para uma análise regional sobre essas alterações. Uma maneira bastante difundida de se avaliar as mudanças climáticas, a nível local e regional, é através da análise dos índices de detecção de mudanças climáticas, criados pela Equipe de Especialista em Detecção, Monitoramento e Índices de Mudanças Climáticas (Expert Team on Climate Change Detection, Monitoring and Índices - ETCCDMI) (SOUZA; AZEVEDO, 2012); e com mais frequência, através da análise de tendências de séries históricas de temperatura do ar e precipitação.

A precipitação e a temperatura do ar são os elementos meteorológicos mais utilizados para avaliar essas mudanças, pois são os mais importantes no campo da climatologia e da hidrologia (GALLEGOS, 2016). A precipitação é uma componente crítica no processo chuva-vazão, com influência direta nas inundações e nos períodos de seca. Enquanto a temperatura do ar exerce um papel relevante na evaporação, transpiração e demanda de água para os as plantas, animais e seres humanos. Ao mesmo tempo em que, tem efeitos significativos no abastecimento de água e sua disponibilidade. Portanto, a identificação do que vem mudando nessas variáveis nos últimos anos, é uma contribuição vital para garantia das atividades socioeconômicas (pecuária, agricultura, turismo e geração de energia), e para fornecer subsídios aos formuladores de políticas públicas e aos técnicos que auxiliam os tomadores de decisão.

As mudanças climáticas estão vinculadas a maior ocorrência de eventos extremos (MARENGO, 2010). Nas regiões que já estão sob domínio contínuo de eventos extremos, como nas regiões áridas e de cerrado, as quais, os eventos de seca e temperaturas elevadas são fenômenos 
comuns, as implicações das mudanças climáticas podem ser mais significativas (IPCC, 2014). O aumento da temperatura nessas regiões pode causar um aumento na evapotranspiração potencial, levando a condições severas de estresse hídrico, comprometendo a segurança alimentar. Consequentemente, nessas regiões onde os eventos extremos já são intensos e/ou frequentes os custos econômicos e sociais deverão aumentar.

O estado do Tocantins é uma dessas regiões que está sob domínio contínuo de eventos extremos, pois o bioma do cerrado ocupa mais de $87,8 \%$ do seu território. Atualmente o Tocantins é o maior produtor de grãos da região Norte do Brasil, sobretudo de soja. Várias pesquisas já foram realizadas sobre a variabilidade e tendência da precipitação ao logos das décadas no Tocantins, mas ainda há uma carência de estudos sobre a tendência temporal da temperatura do ar. Nesse contexto, é necessário conhecer e desenvolver estudos do comportamento dessa variável.

Os testes estatísticos são as principais ferramentas utilizadas para detecção de tendências em séries de observações hidrológicas, com destaque para os classificados como não-paramétricos. Os testes não-paramétricos apresentam uma peculiaridade, de não necessitarem de especificação prévia do modelo populacional, exigindo apenas a independência entre os elementos amostrais (SHADMANI et al., 2012). O teste não-paramétrico mais utilizado na análise de tendências de séries hidro meteorológicas é o teste de Mann-Kendall (MK) (SILVA; SILVA, 2011). O MK também é considerado o teste mais apropriado para analisar mudanças climáticas em séries climatológicas, pois é robusto e de baixa sensibilidade a quebras abruptas em séries temporais (GOOSSENS; BERGER, 1996; YUE et al., 2002; YUE; WANG, 2002; ZAMANI et al., 2016). Por esse motivo o teste de MK vem sendo empregado com frequência em analises de variação temporal das séries ambientais, com objetivos de identificar possíveis evidências de mudanças climáticas (PEREIRA et al., 2017).

Assim, este artigo tem por objetivo detectar a existência de possíveis tendências, com significância estatística, nos eventos extremos temperatura máxima e mínima do ar, em seis municípios do Estado do Tocantins, considerando o período dos anos de 1961 a 2017. Espera-se a partir dessa investigação contribuir com o estado da arte do conhecimento da variabilidade temporal dos extremos de temperatura no Estado do Tocantins, dada a carência de informações associada às projeções de mudanças climáticas globais, às oscilações naturais do clima e ao processo de urbanização.

\section{MATERIAL E MÉTODOS}

\subsection{Dados meteorológicos}

O Estado do Tocantins está localizado a sudeste da Região Norte do Brasil, limita-se com o Maranhão a nordeste, o Piauí a leste, a Bahia a sudeste, o Goiás a sul, Mato Grosso a sudoeste e o Pará a noroeste. O Tocantins abrange uma área de $278.846,9 \mathrm{~km} 2$, inserido entre os paralelos $5^{\circ} 10^{\prime}$ $06^{\prime \prime}$ e $13^{\circ} 27^{\prime} 59^{\prime \prime}$ de latitude sul, e entre os meridianos $45^{\circ} 44^{\prime}$ $46^{\prime \prime}$ e $50^{\circ} 44^{\prime} 33^{\prime \prime}$ de longitude oeste.

Morfologicamente, o Tocantins exibe uma variedade de aspectos, resultante da evolução dos fatores morfoclimáticos e da adaptação das diversidades litológicas e/ou a condicionamentos estruturais a que submeteram. Estes fatores resultaram na presença de diversos níveis altimétricos. Quanto a cobertura vegetal, o cerrado é a cobertura predominante no Tocantins, com um percentual de $87,8 \%$. Três tipos de clima atuam sobre o Tocantins, de acordo com a classificação climática de Thornthwaite; Mather (1955), no oeste do estado situa-se um clima úmido e a leste e extremo norte do estado atuam os climas subúmido e subúmido seco, respectivamente. Nesses climas verificam-se duas estações bem definidas, uma seca (maio e setembro) e a outra chuvosa (outubro e abril). Segundo as Normais Climatológicas (19611990) do INMET, as maiores temperaturas do ar são ocorrem no mês de agosto e menores temperaturas no mês de julho. A temperatura máxima anual do Tocantins varia de $31,1^{\circ} \mathrm{C}$ a $32,9^{\circ} \mathrm{C}$ e a temperatura mínima anual de $19,9^{\circ} \mathrm{C}$ a $21,3^{\circ}$ C. Por outro lado, nas novas Normais Climatológicas (1981-2010) do Instituto Nacional de Meteorologia (INMET), a temperatura máxima anual varia de $31,9^{\circ} \mathrm{C}$ a $33,7^{\circ} \mathrm{C}$ e a temperatura mínima anual de $20,2^{\circ} \mathrm{C}$ a $21,9^{\circ} \mathrm{C}$. De acordo com ambas Normais Climatológicas, as maiores temperaturas máximas anuais foram registradas em Porto Nacional e as menores temperaturas mínima anuais em Taguatinga.

Utilizaram-se dados diários das temperaturas absolutas do ar máxima (Tmáx) e mínima (Tmín) de seis estações meteorológicas convencionais da Rede do INMET, localizadas no estado do Tocantins, disponíveis no Banco de Dados Meteorológicos para Ensino e Pesquisa (BDMEP). Os dados de temperatura do ar foram examinados quanto à qualidade e quanto à homogeneidade. Para preenchimento de falhas de dados de temperatura foi utilizado o cálculo da média dos três dias anteriores, porém no caso de mais de três dias sem dados considerou-se a média entre os três meses de anos anteriores ou posteriores, conforme Penereiro et al. (2012). A série histórica da maioria das estações é superior a 30 anos, entre 1961 a 2017, a exceção da estação de Palmas com período de 1995 a 2017. A cidade de Palmas, capital do Tocantins, foi criada no ano de 1989 e teve a implantação da sua estação meteorológica apenas em outubro de 1993. Nas estações de Pedro Afonso verificou-se ausência de dados de temperatura máxima e mínima no ano de 2017. Nesse mesmo ano as estações de Araguaína e Porto Nacional apresentou ausência de dados de temperatura mínima. A localização geográfica das estações meteorológicas do estado do Tocantins, destacando a irregularidade e a baixa densidade na distribuição das estações meteorológicas no estado é mostrada na Tabela 1.

Tabela 1. Estações meteorológicas utilizadas neste estudo, com as respectivas localizações geográficas, altitude e períodos das séries históricas.

Table 1. Meteorological stations used in this study, with their geographic locations, altitude and periods of historical series.

\begin{tabular}{lllll}
\hline \multicolumn{1}{c}{ Estações } & $\begin{array}{c}\text { Latitude } \\
\left({ }^{\circ}\right)\end{array}$ & $\begin{array}{c}\text { Longitude } \\
\left({ }^{\circ}\right.\end{array}$ & $\begin{array}{c}\text { Altitude } \\
(\mathrm{m})\end{array}$ & Período \\
\hline Araguaína & $-7,20$ & $-48,20$ & 228,5 & $1948-2017$ \\
Pedro Afonso & $-8,96$ & $-48,13$ & 187,0 & $1977-2017$ \\
Palmas & $-10,19$ & $-48,30$ & 280,0 & $1995-2017$ \\
Porto Nacional & $-10,71$ & $-48,41$ & 239,2 & $1975-2017$ \\
Peixe & $-12,01$ & $-48,35$ & 242,5 & $1975-2017$ \\
Taguatinga & $-12,40$ & $-46,41$ & 603,2 & $1961-2017$ \\
\hline
\end{tabular}

As tendências e significância estatística das temperaturas do ar absoluta máxima e mínima, inicialmente foram calculadas para todo o período da série histórica e 
posteriormente para períodos igual ou superior a dez anos. Com o objetivo de verificar o comportamento da temperatura ao longo das últimas décadas. Na Tabela 2 são mostrados os períodos obtidos a partir da divisão das séries históricas de cada estação.

Tabela 2. Divisão das séries históricas de cada estação, em períodos igual ou maior que dez anos para cada.

Table 2. Division of the historical series of each station, in periods equal to or greater than ten years for each.

\begin{tabular}{|c|c|c|c|c|c|}
\hline \multirow[b]{2}{*}{ Estações } & \multicolumn{5}{|c|}{ Período } \\
\hline & $1^{\mathrm{a}}$ & $2^{\mathrm{a}}$ & $3^{a}$ & $4^{a}$ & $5^{\mathrm{a}}$ \\
\hline Araguaína & 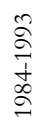 & 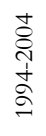 & $\begin{array}{l}\hat{\sigma} \\
\tilde{N} \\
\stackrel{1}{0} \\
\stackrel{0}{0}\end{array}$ & & \\
\hline Pedro Afonso & 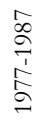 & $\begin{array}{l}\stackrel{2}{\circ} \\
\stackrel{1}{\frac{1}{2}} \\
\stackrel{\infty}{\sigma}\end{array}$ & 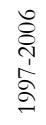 & 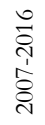 & \\
\hline Palmas & 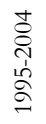 & 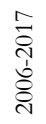 & & & \\
\hline Porto Nacional & 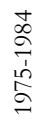 & 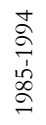 & 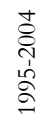 & 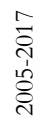 & \\
\hline Peixe & 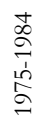 & 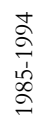 & 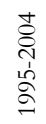 & 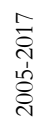 & \\
\hline Taguatinga & 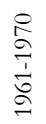 & 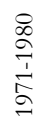 & 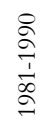 & $\begin{array}{l}8 \\
\stackrel{0}{1} \\
\frac{1}{\sigma} \\
\sigma\end{array}$ & 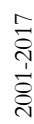 \\
\hline
\end{tabular}

\subsection{Teste de Mann-Kendall (MK)}

Para a análise dos dados, utilizou-se o teste não paramétrico de Mann-Kendall (MANN, 1945; KENDALL, 1975). As avaliações foram feitas na escala anual, através de avaliações da série histórica das temperaturas ao longo do ano (valores extremos anuais) de cada estação meteorológica. O teste de Mann-Kendall (MK) é uma ferramenta estatística recomendada pela Organização Meteorológica Mundial (OMM), eficiente e amplamente utilizada, na avaliação de possíveis tendências em séries temporais de dados ambientais (SILVA et al., 2010; MUSLIH; BLAŻEJCZYK, 2017; BOMBARDI; CARVALHO, 2017).

O teste de MK apresenta duas peculiaridades: ser menos sensível as interrupções abruptas nos dados resultantes de séries temporais não homogêneas Gilbert (1983) e ser mais potente quando se mede as tendências lineares nãoparamétricas (ONOZ; BAYAZIT, 2003). Outra particularidade deste método é a exigência de dados independentes e aleatórios (NEETI; EASTMAN, 2011).

O teste de Mann-Kendall, utilizado para avaliar a tendência de uma série temporal, é calculada pelas Equações (1) e (2)

$$
S=\sum_{i<j} a_{i j}
$$

de modo que:

$$
a_{i j}=\operatorname{sinal}\left(x_{j}-x_{i}\right)=\left\{\begin{array}{l}
+1 \text { se } x_{i}<x_{j} \\
0 \text { se } x_{i}=x_{j} \\
-1 \text { se } x_{i}>x_{j}
\end{array}\right.
$$

em que: considerando a estatística $\mathrm{S}$, é o somatório dos sinais (sinal) da diferença, par a par, de todos valores da série (xi) em relação aos valores em que a ele são futuros (xj).

Quando $\mathrm{n} \geq 10$, a variável $\mathrm{S}$ pode ser comparada com uma distribuição normal, na qual a sua variância $(\operatorname{Var}(\mathrm{S}))$, pode ser obtida através da Equação (3):

$$
\operatorname{Var}(\mathrm{S})=\frac{n(n-1)(2 n+5)-\sum_{i=1}^{q} t_{p}\left(t_{p}-1\right)\left(2 t_{p}+5\right)}{18}
$$

em que: em que tp representa a quantidade de repetições de uma extensão i e q é o número de grupos contendo valores iguais na série de dados em um grupo p. O segundo termo representa um ajuste para dados censurados.

O teste estatístico parametrizado (ZMK) é computado pela Equação (4). A presença de uma tendência estatisticamente significativa é avaliada usando o valor de ZMK. Um valor positivo de ZMK indica um aumento da tendência, quando negativa indica uma tendência decrescente. Para testar a significância da tendência crescente ou decrescente no nível de significância de $\mathrm{p}$, a hipótese nula e rejeitada se o valor absoluto de $Z$ for maior que $Z(1-p / 2)$, obtida da tabela da distribuição normal cumulativa padrão. Neste estudo foram adotados dois níveis de significância: de $\mathrm{a}=0,05$ e a $=0,01$, que corresponde respectivamente a $5 \%$, quando a hipótese nula de não tendência é rejeitada se $|\mathrm{Z}|$ $>1,96$, e a $1 \% \%$, a hipótese a hipótese nula de não tendência é rejeitada se $|Z|>2,57$.

$$
Z_{M K}=\left\{\begin{array}{l}
\frac{s-1}{\sqrt{\operatorname{Var}(s)}} ; \text { se } S>0 \\
0 ; \text { se } S=0 \\
\frac{s+1}{\sqrt{\operatorname{Var}(s)}} ; \text { se } S<0
\end{array}\right.
$$

O teste de Mann-Kendall detecta tendências estatisticamente significativas, mas não fornece estimativas das magnitudes dessas tendências (PORTELA et al., 2011). De acordo com Silva et al. (2010), para estimar a magnitude da inclinação da tendência, através de uma estatística não paramétrica, utiliza-se o estimador estatístico da tendência, também denominado de estimador de inclinação de Sen (SEN, 1968), obtido como Hirsch et al. (1993) através da estatística $\beta$ (Equação 5). O estimador de tendência de Sen seleciona entre todas as linhas de inclinação formadas por cada par de pontos da amostra, aquela correspondente à inclinação mediana possibilita identificar se houve, ou não, mudança de tendência e a magnitude na série.

$$
\beta=\operatorname{mediana}\left[\frac{x_{j}-x_{i}}{(j-i)}\right], \text { para } i<j
$$

em que: xi e xj representam os valores da variável em estudo nos anos i e j. $O$ valor de $\beta$ positivo indica tendência crescente e o valor negativo indica tendência decrescente, respectivamente.

\section{RESULTADOS}

A detecção de tendências das séries temporais de temperatura do ar máxima e mínima, com a devida quantificação, foi realizada através da aplicação do teste não- 
paramétrico de Mann-Kendall e pelo estimador de inclinação de Sen, primeiramente para o período de 1961-2017 e posteriormente para subperíodos maiores que dez anos.

\subsection{Temperatura do ar máxima absoluta}

A média da temperatura máxima absoluta das seis estações meteorológicas que representam o estado do Tocantins, no período analisado, variou de $37,2^{\circ} \mathrm{C}$ a $40,1^{\circ} \mathrm{C}$. Os maiores valores de temperaturas máximas absolutas registradas em cada estação foram, de: $43,0^{\circ} \mathrm{C}(2017) \mathrm{em}$ Palmas; 41, $5^{\circ}$ C (2015) em Peixe; $41,2^{\circ}(2010)$ em Porto Nacional; $40,9^{\circ} \mathrm{C}(2015)$ em Pedro Afonso; $40,0^{\circ} \mathrm{C}(2015)$ em Taguatinga; e $38,6^{\circ} \mathrm{C}$ (2014) em Araguaína. Para todas as estações as maiores temperaturas máximas absolutas, acima de $38^{\circ} \mathrm{C}$, ocorreram com maior frequência a partir da última década. Evidenciando tendência de aquecimento em todas as estações, sendo mais acentuado a partir do final da década de noventa (Figura 1).

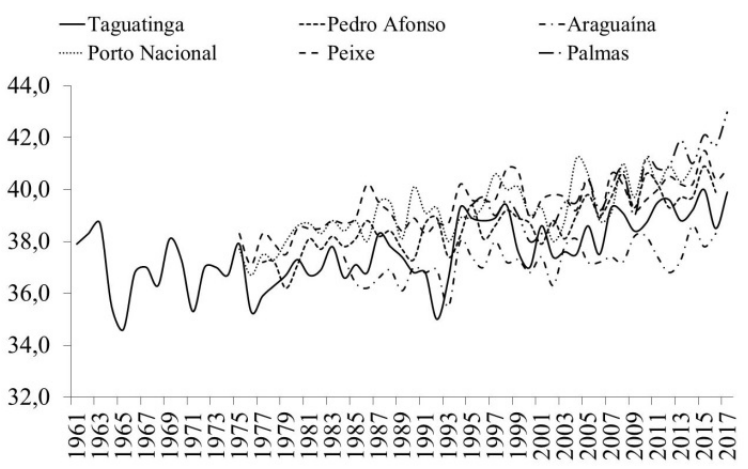

Figura 1. Temperatura do ar máxima absoluta anual das seis estações meteorológicas do estado do Tocantins no período de 1961 a 2017. Figure 1. Maximum annual absolute air temperature of the six meteorological stations in the state of Tocantins from 1961 to 2017.

A Tabela 3 mostra os resultados obtidos a partir da aplicação do teste estatístico de Mann-Kendall, os níveis de significância e as tendências das temperaturas máximas do ar. Quando observamos os resultados das series de temperatura máxima para o Teste de MK para todas as estações, verificamos que houve tendência positiva com significância estatística de $1 \%(\alpha=0,01)$ em todas as estações.

As maiores tendências significativas para as estações de Pedro Afonso e Peixe, com magnitude de inclinação de $0,08^{\circ}$ C ano $^{-1}$ foram obtidas para o período de 1977 a 2017. Por outro lado, a menor tendência e a menor magnitude de inclinação $\left(0,04^{\circ} \mathrm{C}\right.$ ano $\left.{ }^{-1}\right)$ foram obtidas para a estação de Araguaína, no período de 1995-2017. A estação de Palmas foi a que apresentou maior inclinação de $0,16^{\circ} \mathrm{C}$ ano-1, também é a estação que apresenta menor série histórica, de apenas vinte três anos.

As magnitudes das tendências de temperatura do ar máxima absoluta para os níveis de significância estatística adotados nesse estudo, no período igual ou maior que dez anos, revelam que houve pouca variabilidade em relação às temperaturas máxima, com predomínio de tendência positiva, porém sem significância estatística para a maioria das estações (Tabela 4).

A estação de Taguatinga é a que apresenta maior série histórica, de 1961 a 2017, com o total de cinco períodos maior ou igual a dez anos. Apenas o período de 2001 a 2017 da estação de Taguatinga apresentou magnitude de tendência positiva, com significância estatística de $1 \%$, de $0,13^{\circ} \mathrm{C}$ ano ${ }^{1}$ que corresponde a um acréscimo de $2,21^{\circ} \mathrm{C}$ na temperatura máxima. As estações de Pedro Afonso, Porto Nacional e Peixe apresentaram magnitude de tendências positiva, com significância estatística de $1 \%$, apenas para os primeiros períodos, que correspondem respectivamente a 1977-1986, 1975-1984 a 1975-1984. A estação de Araguaína não apresentou magnitude de tendência com significância estatística em nenhum dos períodos. Palmas é a estação que apresenta menor série histórica, com dados de 1995 até 2017. No período de 2005 a 2017 verifica-se magnitude de tendência positiva de $0,23^{\circ}$ C.ano ${ }^{-1} \mathrm{com}$ significância estatística de $5 \%$, que corresponde a um aumento de $2,76^{\circ} \mathrm{C}$ na temperatura máxima em um período de 12 anos.

Tabela 3. Teste de Mann-Kendall e a tendência da temperatura máxima do ar das estações do estado do Tocantins, ao nível de confiança de $\alpha=0,01$ e 0,05, para o período desde 1961 a 2017. Table 3. Mann-Kendall test and the tendency of maximum air temperature of the stations of the state of Tocantins, to the confidence level of $\alpha=0.01$ and 0.05 , for the period from 1961 to 2017.

\begin{tabular}{lccc}
\hline Estações & $\begin{array}{c}\text { Estatística } \\
\text { Padronizada de } \\
\text { Mann-Kendall } \\
\left(\mathrm{Z}_{\mathrm{mk}}\right)\end{array}$ & $\alpha$ & $\begin{array}{c}\text { Estimador } \\
\text { de Sen }(\beta) \\
\left({ }^{\circ} \mathrm{C} \text { ano- }\right.\end{array}$ \\
\hline Araguaína & 3,51 & $\mathrm{~S}^{* *}$ & 0,04 \\
Pedro Afonso & 5,31 & $\mathrm{~S}^{* *}$ & 0,08 \\
Palmas & 4,04 & $\mathrm{~S}^{* *}$ & 0,16 \\
Porto Nacional & 3,77 & $\mathrm{~S}^{* *}$ & 0,07 \\
Peixe & 5,77 & $\mathrm{~S}^{* *}$ & 0,06 \\
Taguatinga & 4,31 & $\mathrm{~S}^{* *}$ & 0,05 \\
\hline
\end{tabular}
= nível de significância estatística a 0,05; ** = nível de significância estatística a 0,01 .

Tabela 4. Magnitude da tendência das temperaturas máximas do ar por período ( ${ }^{\circ} \mathrm{C} /$ período) com nível de confiança $\alpha=0,01$ e 0,05 . Table 4. Magnitude of maximum air temperature trend by period ${ }^{\circ}$ $\mathrm{C} /$ period) with confidence level $\alpha=0.01$ and 0.05 .

\begin{tabular}{lccccc}
\hline \multirow{2}{*}{ Estações } & \multicolumn{5}{c}{ Período } \\
\cline { 2 - 6 } & $1^{\mathrm{a}}$ & $2^{\mathrm{a}}$ & $3^{\mathrm{a}}$ & $4^{\mathrm{a}}$ & $5^{\mathrm{a}}$ \\
\hline Araguaína & $-0,06$ & $-0,06$ & $+0,04$ & & \\
Pedro Afonso & $+0,19^{*}$ & $+0,05$ & $-0,04$ & $+0,01$ & \\
Palmas & $-0,04$ & $+0,23^{* *}$ & & & \\
Porto & $+0,18^{* *}$ & 0,00 & 0,00 & $+0,09$ & \\
Peixe & $+0,11^{* *}$ & $-0,03$ & $-0,04$ & $+0,09$ & \\
Taguatinga & $-0,07$ & $+0,04$ & $+0,06$ & $+0,20$ & $+0,13$ \\
\hline
\end{tabular}

* = nível de significância estatística a 0,$05 ; * *=$ nível de significância estatística a 0,01 .

Todas as estações apresentaram tendência positiva e com significância estatística de $1 \%(\alpha=0,01)$ (Tabela 3). A estação de Palmas registrou a maior inclinação da tendência, correspondendo um aumento da taxa de temperatura máxima absoluta de $3,68^{\circ} \mathrm{C}$ no período de 23 anos. A estação de Pedro Afonso também apresentou aumento da taxa de temperatura máxima, com inclinação de tendência de $3,20^{\circ} \mathrm{C}$ em 40 anos. A menor taxa de aumento da temperatura, com inclinação de tendência de $1,32^{\circ} \mathrm{C}$ em 33 anos foi observado na estação de Araguaína. Na estação de Taguatinga também se observou inclinação da tendência de temperatura superior a $3,0^{\circ} \mathrm{C}$, porém para um período de tempo superior a 50 anos. 
Tabela 5. Magnitude das tendências das temperaturas máxima do ar ao logo do período da series de dados de cada estação.

Table 5. Magnitude of the trends of the maximum air temperatures to the logo of the period of the data series of each station.

\begin{tabular}{lcc}
\hline Estações & $\begin{array}{c}\text { Inclinação da tendência } \\
\left({ }^{\circ} \mathrm{C} / \text { periodo) }\right.\end{array}$ & $\begin{array}{c}\text { Período } \\
\text { (anos) }\end{array}$ \\
\hline Araguaína & $1,32^{\circ} \mathrm{C}$ & 33 \\
Pedro Afonso & $3,20^{\circ} \mathrm{C}$ & 40 \\
Palmas & $3,68^{\circ} \mathrm{C}$ & 23 \\
Porto Nacional & $3,01^{\circ} \mathrm{C}$ & 43 \\
Peixe & $2,58^{\circ} \mathrm{C}$ & 43 \\
Taguatinga & $2,85^{\circ} \mathrm{C}$ & 57 \\
\hline
\end{tabular}

\subsection{Temperatura do ar mínima absoluta}

A média da temperatura mínima absoluta do Tocantins, para as seis estações meteorológicas consideradas nesse estudo, é de $15,1{ }^{\circ} \mathrm{C}$. Em Araguaína e Porto Nacional verificam-se as menores médias de temperaturas mínimas absolutas, de respectivamente $13,2^{\circ} \mathrm{C}$ e $14,1^{\circ} \mathrm{C}$. Nas demais estações as médias das temperaturas mínima absoluta ficam em torno de $16,0^{\circ} \mathrm{C}$ e ocorrem com mais constância a partir de 2007 (Figura 2).

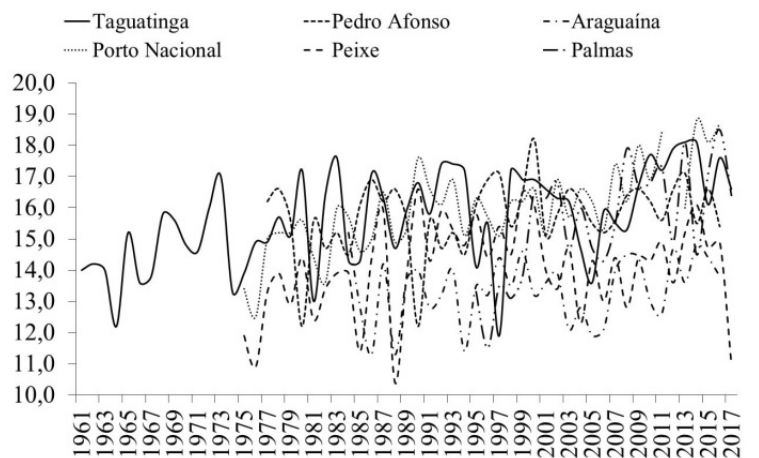

Figura 2. Temperatura do ar mínima absoluta anual das seis estações meteorológicas do estado do Tocantins no período de 1961 a 2017. Figure 2. Temperatura do ar mínima absoluta anual das seis estações meteorológicas do estado do Tocantins no período de 1961 a 2017.

Tabela 6. Teste de Mann-Kendall e a tendência da temperatura mínima do ar das estações do estado do Tocantins, ao nível de confiança de $\alpha=0,01$ e 0,05, para o período desde 1961 a 2017.

Table 6. Mann-Kendall test and the tendency of the minimum air temperature of the stations of the state of Tocantins, to the confidence level of $\alpha=0.01$ and 0.05 , for the period from 1961 to 2017.

\begin{tabular}{lccc}
\hline Estações & $\begin{array}{c}\text { Estatística } \\
\text { Padronizada } \\
\text { de } \\
\text { Mann-Kendall } \\
\left(\mathrm{Z}_{\mathrm{mk}}\right)\end{array}$ & $\alpha$ & $\begin{array}{c}\text { Estimador de } \\
\text { Sen }(\beta) \\
\left({ }^{\circ} \mathrm{C} \text { ano- }{ }^{-1}\right)\end{array}$ \\
\hline Araguaína & 1,27 & $\mathrm{NS}$ & 0,02 \\
Pedro Afonso & 1,47 & $\mathrm{NS}$ & 0,03 \\
Palmas & 3,33 & $\mathrm{~S}^{* *}$ & 0,18 \\
Porto Nacional & 5,10 & $\mathrm{~S}^{*}$ & 0,10 \\
Peixe & 1,91 & $\mathrm{NS}$ & 0,03 \\
Taguatinga & 3,56 & $\mathrm{~S}^{*}$ & 0,05 \\
\hline S = estatisticamente significativo; $\mathrm{NS}=$ não significativo estatisticamente; * \\
$=$ nível de significância estatística a 0,$05 ; * *=$ nível de significância estatística \\
a $0,01$.
\end{tabular}

Notam-se valores positivos de ZMK em todas as estações, indicando tendência de aumento da temperatura mínima absoluta (Tabela 4). Porém com significância estatística de $1 \%(\alpha=0,01)$, apenas para as estações de Palmas, Porto Nacional e Taguatinga (Tabela 6). Esses resultados confirmam a ocorrência de tendência de aquecimento nesses municípios. As estações de Araguaína, Pedro Afonso e Peixe apresentaram peculiaridade, não apresentaram significância estatística e foram as estações que apresentaram os menores valores de ZMK. Observa-se também que as estações de Palmas e Pedro Afonso, que apresentaram as maiores tendências significativas, foram as que apresentaram maior inclinação. No período de 23 anos, Palmas sofreu aumento de $0,18^{\circ} \mathrm{C}$ ano ${ }^{-1}$ na temperatura mínima. Já Pedro Afonso o aquecimento foi de $0,10^{\circ} \mathrm{C}$ por ano, mas em um período maior, de 40 anos.

As observações de um modo geral revelam que houve pouca variabilidade em relação às temperaturas mínima. Nas tendências de temperatura mínima predominaram tendências positivas, porém não houve significância para os valores (Tabela 7). A estação de Taguatinga é a que apresenta maior série histórica, de 1961 a 2017, com o total de cinco períodos maior ou igual a dez anos. Apenas o período de 2001 a 2017 da estação de Taguatinga apresentou magnitude de tendência positiva, com significância estatística de $5 \%$, de $0,19^{\circ} \mathrm{C}$ ano ${ }^{1}$ que corresponde a um acréscimo de $3,23^{\circ} \mathrm{C}$ na temperatura mínima em 17 anos. As estações de Pedro Afonso e Palmas apresentaram magnitude de tendências positiva, com significância estatística de 1\%, apenas para os períodos de 1987-1996 e 1995-2004, respectivamente. As estações de Araguaína, Porto Nacional e Peixe, não apresentou magnitude de tendência com significância estatística em nenhum dos períodos. Palmas no período de 1995 a 2004 a magnitude de tendência positiva de $0,46^{\circ} \mathrm{C}$ ano ${ }^{-1} \mathrm{com}$ significância estatística de $5 \%$, que corresponde a um aumento de $4,6^{\circ} \mathrm{C}$ na temperatura mínima em um período de 10 anos.

Tabela 7. Magnitude da tendência das temperaturas mínimas do ar por período $\left({ }^{\circ} \mathrm{C} /\right.$ período $)$ com nível de confiança $\alpha=0,01$ e 0,05 .

Table 7. Magnitude of the trend of minimum air temperatures per period $\left({ }^{\circ} \mathrm{c} /\right.$ period) with confidence level $\alpha=0.01$ and 0.05 .

\begin{tabular}{cccccc}
\hline \multirow{2}{*}{ Estações } & $1^{\mathrm{a}}$ & $2^{\mathrm{a}}$ & $3^{\mathrm{a}}$ & $4^{\mathrm{a}}$ & $5^{\mathrm{a}}$ \\
\cline { 2 - 6 } & $+0,01$ & $+0,05$ & $+0,15$ & & \\
Araguaína & $+0,03$ & $+0,34^{*}$ & $+0,02$ & $+0,07$ & \\
Pedro Afonso & $+0,46^{*}$ & $+0,15$ & & & \\
Palmas & $+0,22$ & $+0,14$ & $+0,05$ & $+0,20$ & \\
Porto Nacional & $+0,22$ & $+0,31$ & $-0,25$ & $+0,01$ & \\
Peixe & $+0,0,19^{*}$ \\
Taguatinga & $+0,18$ & $+0,10$ & $+0,15$ & $-0,05$ & $+0,1)^{*}$
\end{tabular}

As magnitudes das tendências de temperatura mínima do ar para as estações do estado do Tocantins no respectivo período da série histórica são mostradas na Tabela 8. A maior inclinação de tendência positiva foi obtida para a estação de Palmas, com acréscimo $4,14^{\circ} \mathrm{C}$ na temperatura mínima no período de 23 anos, seguido por Pedro Afonso com inclinação de tendência positiva de $3,28^{\circ} \mathrm{C}$, porém em um período de tempo maior que 41 anos. A menor inclinação de tendência DA temperatura mínima foi observada para a estação de Araguaína, de $0,66^{\circ} \mathrm{C}$, em um período de 33 anos. $\mathrm{Na}$ estação de Taguatinga também se observa tendência de aumento de $2,85^{\circ} \mathrm{C}$ na temperatura mínima do ar para um período de 57 anos. 
Tabela 8. Magnitude das tendências das temperaturas mínima do ar ao longo do período da series de dados de cada estação.

Table 8. Magnitude of the minimum air temperature trends over the period of the data series of each season.

\begin{tabular}{lcc}
\hline \multicolumn{1}{c}{ Estações } & $\begin{array}{c}\text { Inclinação da tendência } \\
\left({ }^{\circ} \mathrm{C} / \text { periodo) }\right.\end{array}$ & $\begin{array}{c}\text { Período } \\
\text { (anos) }\end{array}$ \\
\hline Araguaína & $0,66^{\circ} \mathrm{C}$ & 33 \\
Pedro Afonso & $1,20^{\circ} \mathrm{C}$ & 40 \\
Palmas & $4,14^{\circ} \mathrm{C}$ & 23 \\
Porto Nacional & $3,28^{\circ} \mathrm{C}$ & 43 \\
Peixe & $1,29^{\circ} \mathrm{C}$ & 43 \\
Taguatinga & $2,85^{\circ} \mathrm{C}$ & 57 \\
\hline
\end{tabular}

\section{DISCUSSÃO}

Os resultados das análises realizadas indicam claramente um aumento da temperatura, com aquecimento, nas seis estações registradas. A variação da temperatura do ar é atribuída à energia em a forma de calor sensível presente no ar. As maiores temperaturas máximas foram observadas nas estações de Palmas e Taguatinga, no período de 2000 a 2017 (Tabela 4). Também é observável que os valores mais baixos nas temperaturas máximas foram registrados nas décadas de 1970 e 1980, revelando aumento nas temperaturas diurnas ao longo dos anos. Segundo Andrade et al. (2012) o aumento das temperaturas diurnas pode ser indicativo de mudança no uso da terra, onde superfícies de maior albedo ou menor emissividade são substituídos por outras superfícies que apesar de apresentarem maior capacidade de absorção e emissão, substitui o fluxo de calor latente pelo fluxo de calor sensível. A remoção ou redução da superfície com vegetação, também é outro fato que pode promover o aumento da temperatura do ar, pois diminui a quantidade de energia usada como calor latente de evapotranspiração.

Os resultados também indicam claramente um aumento nas temperaturas máximas e mínimas, nas seis estações, independentemente da altitude. Nota-se que embora o município de Palmas tenha uma população de 228.332 pessoas, que é 19 vezes maior do que as populações de Pedro Afonso, com 11.539 pessoas, as tendências e as inclinações de temperatura dessas duas estações são muito semelhantes. Palmas também sofreu impacto de perda de área. No ano de 2001 a sua área era da ordem de $2.026 .829,78 \mathrm{~km}^{2}$, por motivo de enchimento do Lago da Usina Hidroelétrica do Lajeado, ocorreu uma supressão de área em torno de aproximadamente $10 \%$, restando então $1.814 .304,94 \mathrm{~km}^{2}$. Destaca-se que os maiores extremos de temperaturas máximas e mínimas do ar foram registrados a partir de meados da década de 1990, com os maiores incrementos ocorridos principalmente na última década o que indica aumento das temperaturas diurnas. Os maiores aumentos de temperatura máxima ocorreram nas estações de Pedro Afonso, Palmas, Porto Nacional e Peixe. Os menores foram observados nas estações de Araguaína e Taguatinga.

$\mathrm{Na}$ estação de Palmas verificou-se aumento na temperatura mínima foi mais acentuado do que o aumento da temperatura máxima. Resultados semelhantes foram observados por outros pesquisadores (JUNG et al., 2002; LADOCHY et al., 2007; VOSE et al., 2005; TAO et al., 2014; YANEZ; VILLARROEL, 2016), mostrando que, embora exista uma alta variabilidade regional no aumento da temperatura, a mesma tendência de um aumento é evidente em diferentes partes do globo. A variação da temperatura mínima do ar ocorre devido à energia em trânsito da superfície da terra para a atmosfera na forma de calor sensível, resultando em uma redução dessas trocas, aumentando consequentemente o número de noites mais "quentes".

Tendências parecidas foram encontradas por Penereiro et al. (2018), para os biomas do Cerrado e Amazônia, ao investigarem a tendências de temperatura do ar e precipitação nas quatro estações do ano e diferentes biomas. Ferreira et al. (2015) identificaram tendências significativas de aumento da temperatura máxima anual, para a cidade de Juiz de Fora MG, usando a série histórica de 1972 a 2014. Em Uberaba MG também foi encontrada tendências positivas de temperatura máxima, indicando que esse município está sofrendo elevação das temperaturas máximas ao longo dos anos (PEREIRA et al., 2017). Gallegos (2016) encontrou tendências ascendentes da temperatura do ar máxima e mínima em Chiapas no México. Yanez; Villarroel (2016) também observaram aumento da temperatura máxima do ar na região central do Chile. Muslih \& Błażejczyk ao avaliarem as temperaturas extremas no Iraque, no período de 1941 a 2013, verificaram que a temperaturas máxima do ar da maioria das estações apresentaram tendências ascendentes mais fortes, indicando que estão sofrendo processo de aquecimento. O documento do IPCC (2007) destaca como a principal razão para o aumento da temperatura do ar, as mudanças no uso da terra.

\section{CONCLUSÕES}

Em todas as seis estações verificou-se tendência positiva estatisticamente significante, ao nível de confiança de 99\%, nos extremos anuais de temperatura máxima.

As temperaturas mínimas apresentaram tendência positiva, ao nível de significância estatística de $1 \%$, apenas nas estações de Palmas, Porto Nacional e Taguatinga. Nas demais estações as tendências de temperatura são positivas, mas sem significância estatística.

As maiores tendências de acréscimo da temperatura máxima ocorreram em Palmas e Peixe com uma inclinação de $0,16^{\circ} \mathrm{C}$ ano ${ }^{-1}$ e $0,06^{\circ} \mathrm{C}_{\text {ano }}{ }^{-1}$, respectivamente. As maiores taxas de crescimento da temperatura mínima foram registradas nas estações de Palmas, com uma inclinação de $0,18^{\circ} \mathrm{C}_{\text {ano }}{ }^{-1}$, seguido por Porto Nacional, com $0,10^{\circ} \mathrm{C}$ ano1.

As evidencias de aumento da temperatura do ar máxima e mínima são mais evidentes a partir da década de 90 , com a taxa anual de aumento na temperatura do ar mínima superior as máxima, indicando maior retenção de calor durante a noite.

\section{REFERÊNCIAS}

ANDRADE, C.; LEITE, S.; SANTOS J. A. Temperature extremes in Europe: overview of their driving atmospheric patterns. Natural Hazards and Earth System Sciences, v. 12, n. 5, p. 1671-1691, 2012. DOI: https://dx.doi.org/10.5194/nhess-12-1671-2012

BOMBARDI, R. J.; CARVALHO, L. M. V. Práticas simples em análises climatológicas: uma Revisão. Revista Brasileira de Meteorologia, São José dos Campos, v. 32, n. 3, p. 311-320, 2017. DOI: http://dx.doi.org/10.1590/0102-77863230001

FERREIRA, C. C. M.; BATISTA, G. M. F.; VIANNA, Y. C. G. Variações ou tendências climáticas: um estudo com as 
séries de temperatura do ar para a cidade de Juiz de ForaMG. Revista de Ciências Humanas, Viçosa, v. 15, n. 2, p. 414-433, 2015.

GALLEGOS, J. A. F. Índices de cambio climático en la cuenca del río Sabinal, Chiapas, Mexico. Aqua-LAC, v. 8, n. 2, p. 36-41, 2016.

GILBERT, R. O. Statistical methods for environmental pollution monitoring. New York: Van Nostrand Reinhold, 1983. 320p.

GOOSSENS, C; BERGER, A. Annual and seasonal climatic variations over the northern hemisphere and Europe during the last century. Annales Geophysicae, Berlin, v.4, n. B4, p. 385-400. 1986.

HIRSCH, R. M., HELSEL, D. R., COHN, T. A., \& GILROY, E. J. Statistical Analysis of Hydrologic Data. In: MAIDMENT, D. R. (Ed.). Handbook of Hydrology. New York: McGraw-Hill, In. 1993. cap 17. p. 17.1-17.55.

INMET_INSTITUTO NACIONAL DE METEOROLOGIA. Banco de Dados Meteorológicos para Ensino e Pesquisa. Disponível em:

<http://www.inmet.gov.br/portal/index.php?r=bdmep /bdmet>. Acesso em: 02 Mai. 2018.

IPCC_INTERGOVERNMENTAL PANEL ON CLIMATE CHANGE. Contribution of Working Group I to the Fourth Assessment Report of Intergovernmental Panel on Climate Change. Cambridge: Cambridge University Press, 2007. 976 p.

IPCC_ INTERGOVERNMENTAL PANEL ON CLIMATE CHANGE. Climate Change 2014: Synthesis Report. Contribution of Working Groups I, II and III to the Fifth Assessment Report of the Intergovernmental Panel on Climate Change [Core Writing Team, R.K. Pachauri and L.A. Meyer (eds.)]. Geneva: IPCC, 2014. 151 p.

JUNG, H. S.; CHOI, Y.; OH, J.-H.; LIM, G.-H. Recent trends in temperature and precipitation over South Korea. International Journal Climatology, v. 22, n. 11, p. 1327-1337, $2002 . \quad$ DOI: https://dx.doi.org/10.1002/joc.797

KENDALL, M. G. Rank Correlation Methods. 2 ed. Hardcover: London. 1955. 202 p.

LADOCHY, S.; MEDINA, R.; PATZERT, W. Recent California climate variability: Spatial and temporal patterns in temperature trends. Climate Resource, v. 33, $\begin{array}{llll}\text { n. } 2, \quad \text { p. } 159-169, & 2007 . & \end{array}$ https://dx.doi.org/10.3354/cr033159

MANN, H. B. Non-parametric tests against trend. Econometria, v. 13, p. 245-259, 1945.

MARENGO, J. A. Mudanças Climáticas, Condições Meteorológicas Extremas e Eventos Climáticos no Brasil. In: FBDS (org) Mudanças Climáticas Eventos Extremos no Brasil. p: 05-19. FDBS \& LLOYD’S. 2010.

MUSLIH, K. D, BLAZEJCZYK, K. The inter-annual variations and the long-term trends of monthly air temperatures in Iraq over the period 1941-2013. Theoretical and Applied Climatology, Wien, v. 130, p. 583-596, 2017.2 DOI: https://dx.doi.org/10.1007/s00704-016-1915-6

NEETI, N; EASTMAN, J.R. A Contextual Mann-Kendall Approach for the Assessment of Trend Significance in Image Time Series. Transactions in GIS, Cambridge, v.
15, n. 5, p. 599-611, 2011. DOI: https://dx.doi.org/10.1111/j.1467-9671.2011.01280.x

ÖNÖZ, B; BAYAZIT, M. C. The power of statistical tests for trend detection. Turkish Journal of Engineering and Environmental Sciences, v. 27, p. 247-251, 2003.

PENEREIRO, J. C.; BADINGER, A.; MACCHERI, N. A.; MESCHIATTI, M. C. Distribuições de Tendências Sazonais de Temperatura Média e Precipitação nos Biomas Brasileiros. Revista Brasileira de Meteorologia, v. 33, n. 1, p. 97-113, 2018. DOI: https://dx.doi.org/10.1590/0102-7786331012

PEREIRA, S. M. M.; EMILIANO V. M.; SANCHES, F. O.; FERREIRA, R. V. Temperaturas extremas e as mudanças climáticas em Uberaba (MG): primeiros apontamentos. Os Desafios da Geografia Física na Fronteira do Conhecimento, Campinas, v. 1, p. 1737-1746, 2017. DOI: https://dx.doi.org/10.20396/sbgfa.v1i2017.2299

PORTELA, M. M.; QUINTELA, A. C.; SANTOS, J. F.; VAZ. C; MARTINS, C. Tendências em séries temporais de variáveis hidrológicas. Associação Portuguesa dos Recursos Hídricos, Lisboa, v. 32, p. 43-60, 2011.

SEN, P. K. Estimates of the Regression Coefficient Based on Kendall's Tau. Journal of the American Statistical Association, New York, v. 63, n. 324, p. 1379-1389, 1968.

SHADMANI, M.; MAROFI, S.; ROKNIAN, M. Trend Analysis in Reference Evapotranspiration Using MannKendall and Spearman's Rho Tests in Arid Regions of Iran. Water Resources Manage, v. 26, p. 211-224, 2012.

SILVA, D. F; SILVA, R. A. Uso do teste de Mann-kendall para detecção de tendências climáticas comparativas entre regiões cearenses. In: Simpósio Internacional de Climatologia, 4., 2011, Rio de Janeiro. Anais... Rio de Janeiro: SBMET, 2011. não paginado.

SILVA, R. A.; SILVA, V. P. R.; CAVALCANTI, E. P.; SANTOS, D. N. Estudo da variabilidade da radiação solar no Nordeste do Brasil. Revista Brasileira de Engenharia Agrícola e Ambiental, Campina Grande, v. 14 , n. 5, p. 501-509, 2010. DOI: http:/ /dx.doi.org/10.1590/S1415-43662010000500007

TAO, H.; FRAEDRICH, K.; MENZ, C.; ZHAI, JIANQING. Trends in extreme temperature indices in the Poyang Lake Basin, China. Stoch Environ Res Risk Assess, v. 28, p. 1543-1553, 2014. DOI: https://dx.doi.org/10.1007/s00477-014-0863-x

THORNTHWAITE, C. W.; MATHER, J. R. The water balance. New Jersey: Drexel Institute of Technology, 1955. 104 p. (Publications in Climatology, v. 8, n. 1)

VOSE, R. S.; EASTERLING, D. R.; GLEASON, B. Maximum and minimum temperature trends for the globe: an update through 2004. Geophysics Resource Letter, v. 32, n. 23, 2005. DOI: https://dx.doi.org/10.1029/2005GL024379

YUE, S.; PILON, P.; CAVADIAS, G. Power of the MannKendall and Spearman's rho tests for detecting monotonic trends in hydrological series. Journal of Hydrology, Amsterdam, v. 264, n. 1-4, p. 254-271, march 2002. DOI: https://dx.doi.org/10.1016/S00221694(01)00594-7

YUE S.; WANG C. Applicability of prewhitening to eliminate the influence of serial correlation on the MannKendall test. Water Resources Research, Washington, v. 38 , n. 6 , p. 1068, 2002. DOI: https://dx.doi.org/10.1029/2001WR000861 
Silva et al.

ZAMANI, R., R. MIRABBASI, S. ABDOLLAHI, D. JHAJHARIA. Streamflow trend analysis by considering autocorrelation structure, long-term persistence, and Hurst coefficient in a semi-arid region of Iran.
Theoretical and Applied Climatology, Wien, v. 129, p. 33-45, 2017. DOI: https://doi.org/10.1007/s00704-016$1747-4$ 